\title{
Danos de moscas-das-frutas (Diptera, Tephritidae) em citros, manejados no sistema orgânico de produção
}

\author{
Fernando Felisberto da Silva ${ }^{1}$, Luíza Rodrigues Redaelli², Rafael Narciso Meirelles ${ }^{3}$, Fábio Kessler Dal Soglio ${ }^{4}$ \\ http://dx.doi.org/10.1590/0034-737X201461050006
}

\section{RESUMO}

As moscas-das-frutas são as principais pragas da fruticultura mundial. Consideradas chaves para a produção de citros, torna-se necessário o seu monitoramento, visando a evitar os danos diretos. O experimento teve como objetivos conhecer a variação populacional de Anastrepha fraterculus e a relação de sua população com danos em pomares orgânicos de Citrus sinensis, cultivar Céu e de C. sinensis x Citrus reticulata tangor 'Murcott'. Os dados foram coletados em 2003 e 2004 durante o período de maturação dos frutos, na região do vale do Caí, RS, Brasil. O número de moscas-das-frutas foi registrado, semanalmente, por meio de armadilhas McPhail, contendo suco de uva, a 25\%. Danos aos frutos foram determinados pela razão entre frutos sadios e frutos danificados pela mosca. Registros meteorológicos de temperatura, umidade relativa e precipitação pluviométrica foram obtidos, em estação meteorológica distante $30 \mathrm{~km}$ das áreas experimentais. Verificou-se que, em condições ideais de precipitação pluvial, maiores foram as populações de A. fraterculus, espécie predominante na região. A população estimada capaz de causar danos aos frutos variou de acordo com o cultivar, sendo a laranjeira 'Céu' a mais susceptível. Os maiores picos populacionais ocorrem na fase de mudança de coloração dos frutos. Porém, na fase de maturação, as moscas causaram os maiores danos, dada a intolerância dos frutos ao ataque. Conclui-se que a infestação dos frutos de citros por A. fraterculus está relacionada com espécie e cultivar e com fatores climáticos, principalmente com a precipitação pluvial. O monitoramento constante da população de mosca-das-frutas é importante na determinação da infestação na colheita.

Palavras-chave: Anastrepha fraterculus, monitoramento, dados meteorológicos, agricultura orgânica, danos aos frutos de citros.

\section{ABSTRACT}

\section{Damage caused by fruit flies (Diptera, Tephritidae) on citrus under organic production}

Fruit flies (Diptera, Tephritidae) are the main pests of horticulture worldwide. Because they are considered key pests in citrus production, it is necessary to monitor them to avoid the direct damage. The objective of this experiment was to evaluate the population variation of Anastrepha fraterculus and the relationship of its population with damage in organic orchards of orange "Céu" cultivar and tangor (C. sinensis x Citrus reticulata) cv "Murcott". Data were collected over the maturation period, from January 2003 to September 2004, in the Caí Valley region, State of Rio Grande do Sul, Brazil. The number of fruit flies was recorded weekly by McPhail traps containing grape juice at

Recebido para publicação em 18/10/2012 e aprovado em 06/06/2014.

${ }^{1}$ Engenheiro-Agrônomo, Doutor. Universidade Federal do Pampa, Curso de Agronomia, Avenida Luiz Joaquim de Sa Brito, s/n, Campus Itaqui, 97.650-000, Itaqui, Rio Grande do Sul, Brasil. fernando.silva@unipampa.edu.br (autor para correspondência).

${ }^{2}$ Engenheira-Agrônoma, Doutora. Universidade Federal do Rio Grande do Sul, Faculdade de Agronomia, Avenida Bento Gonçalves, 7712, Campus Agronomia, $91.540-000$, Porto Alegre, Rio Grande do Sul, Brasil. lureadel@ufrgs.br

${ }^{3}$ Engenheiro-Agrônomo, Mestre. Universidade Federal do Rio Grande do Sul, Faculdade de Agronomia, Avenida Bento Gonçalves, 7712, Campus Agronomia, 91.540-000, Porto Alegre, Rio Grande do Sul, Brasil. rafael.meirelles@ufrgs.br

${ }^{4}$ Engenheiro-Agrônomo, Doutor. Universidade Federal do Rio Grande do Sul, Faculdade de Agronomia, Avenida Bento Gonçalves, 7712, Campus Agronomia, 91.540-000, Porto Alegre, Rio Grande do Sul, Brasil. fabiods@ufrgs.br 
$25 \%$. Damageto the fruits were determined by the ratio between healthy fruits and fruit damaged by the flies. Meteorological records of temperature, relative humidity and rainfall were obtained from meteorological station $30 \mathrm{~km}$ away from the experimental areas. It was found that larger populations of A. fraterculus, the predominant species in the region, were found under ideal conditions of rainfall. The estimatedpopulation of fruit flies capable of causing damage to fruits varied according to the cultivar, in which orange cv 'Céu' was the most susceptible. The highest population peaks were found at the color changing phase of fruits. However, at the phase of fruit maturity, flies caused the greatest damage due to the fruits intolerance to the attack. It was concluded that the infestation of citrus fruits by A. fraterculus is related to the species, the cultivar and climatic factors, especially rainfall. Constant monitoring of fruit fly population is important in determining the infestation at harvest.

Key words: Anastrepha fraterculus, damages on citrus fruits, meteorological data, organic agriculture,sampling.

\section{INTRODUÇÃO}

No Brasil, os principais gêneros de moscas-das-frutas são Anastrepha Schiner 1868 e Ceratitis McLeay 1829; deste último, apenas a espécie Ceratitis capitata (Wiedemann, 1824) (Diptera: Tephritidae) é relatada no país (Zucchi, 2000). O gênero Anastrepha, originário do continente sul-americano (Aluja, 1994), é o de maior importância econômica e engloba grande número de espécies, que podem atacar diversas frutíferas de regiões tropicais e subtropicais (Sequeira et al., 2001).

O monitoramento das espécies de moscas-das-frutas é realizado, principalmente, por atrativos alimentares, como sucos de frutas, usados em armadilhas tipo McPhail, ou adaptadas, como garrafas de polietileno de dois litros (Salles, 1995). Atrativo considerado padrão é a proteína hidrolisada, podendo ser utilizado, com a mesma eficiência, suco de uva, a 25\% (Scoz et al., 2006).

Estudos regionalizados sobre ocorrência das moscas-das-frutas, em diferentes frutíferas, são de grande importância para o entendimento de sua distribuição geográfica e para adoção de medidas mitigadoras, considerados sua variação populacional e seu potencial de danos. Variações populacionais das moscas-das-frutas, apesar de não ocorrerem de forma padronizada e definida, podem estar ligadas a fatores climáticos e à disponibilidade ou adequabilidade de hospedeiros (Salles, 1995; Canesin \& Uchôa-Fernandes, 2007; Alberti et al., 2012). Essa variação implica, diretamente, a magnitude dos danos aos frutos e sua detecção é de grande importância para adoção de medidas de redução populacional do inseto, em momento adequado.

Este estudo foi desenvolvido com o objetivo de conhecer a variação populacional das moscas-das-frutas, verificar sua relação com fatores climáticos e o dano aos frutos, de duas espécies de citros, na região do vale do rio Caí, RS.

\section{MATERIAL E MÉTODOS}

O experimento foi realizado em dois pomares de citros Citrus sinensis (L.) Osb. cv. Céu, nos municípios

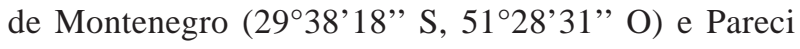
Novo (29³7'22"' S, 51 24 '23' 'O), e dois de Citrus reticulata Blanco x C. sinensis (L.) Osb., tangor 'Murcott', em Montenegro (2941'12' S, 51³1'50' O; 29॰40'12”' S, 51³2'19”' O), região do vale do rio Caí, Rio Grande do Sul. Os pomares tinham entre 8 e 12 anos e eram manejados no sistema orgânico de produção certificado, sem controle de moscas-das-frutas. Amostragens foram realizadas, em 2003 e 2004, de janeiro a maio, para a laranjeira 'Céu', e de maio a setembro, para tangor 'Murcott'.

Armadilhas McPhail com aproximadamente $200 \mathrm{~mL}$ de suco de uva, a $25 \%$, dispostas nos pomares em transecto (leste-oeste) e distanciadas $15 \mathrm{~m}$ entre si e a 1,5 m de altura, foram usadas. Uma armadilha foi instalada para cada quinze plantas, ficando seis e quatro nos pomares de laranjeira e seis e cinco nos de tangoreiro 'Murcott', respectivamente. As amostragens foram semanais, o conteúdo de cada armadilha recolhido e o atrativo trocado. Os tefritídeos coletados foram mantidos em álcool $70 \%$ e separados em laboratório.

A coloração dos frutos foi avaliada visualmente, atribuindo-se as categorias de cores dada pelo software Munsell Conversion versão 4.01 (DG Colour Ltd., 1996): I - verde (2.5G 7/10); II - verde- amarelado ou mudança de coloração (5GY 8.5/10) e III - amareloalaranjado ou maturação (5Y 8/8).

Para a avaliação dos danos, três plantas foram marcadas, com fitas plásticas, no transecto das armadilhas, excluídas aquelas com armadilha. Nessas plantas, foi efetuada contagem prévia dos frutos. Semanalmente, foram recolhidos e contados os frutos caídos, que foram examinados externa e internamente, para verificação de sinais de puncturas e presença de ovos ou lar- 
vas e determinação da provável causa da queda. Em cada ocasião de amostragem, foi calculado o percentual de frutos danificados, por planta, até a época de colheita.

Os frutos recolhidos foram acondicionados em recipientes plásticos com areia umedecida, tampados com tecido tipo voile e mantidos em condição ambiente. Decorridos 20 dias, a areia foi peneirada e os pupários transferidos para caixas gerbox, mantidas em câmara climatizada $\left(25 \pm 1^{\circ} \mathrm{C}\right.$; fotofase 12 horas $)$. A identificação das moscas foi baseada em Zucchi (2000). Os vouchers-specimens foram depositados no Laboratório de Biologia, Ecologia e Controle Biológico, da Universidade Federal do Rio Grande do Sul, em Porto Alegre, RS.

A estimativa do nível de dano foi baseada na análise de regressão linear, entre o número de moscas/armadilha/dia e os danos computados (Pedigo, 1996; Paiva, 2004). O número de moscas, por armadilha, por dia (MAD), capaz de causar dano conhecido (d) de $1 \%$, foi estimado por meio da equação obtida, sendo: $\mathrm{Y}=\mathrm{a}+\mathrm{bx}$; $\mathrm{Y}=$ dano, estabelecido em $1 \%$ da produção $(\mathrm{d})$; $\mathrm{a}=$ constante linear; $\mathrm{Y}=\mathrm{a}$, quando o número de moscas/armadilha/dia for igual a zero; $b=$ dano correspondente a cada mosca por armadilha para ocasionar $\mathrm{Y}(\mathrm{d}) ; \mathrm{x}=$ número de moscas/armadilha/dia (MAD).

A associação entre MAD e danos foi realizada por tentativas, agrupando-se os períodos de picos populacionais e de danos causados aos frutos, até a obtenção de significância na associação. Esse procedimento foi adotado sempre que os danos não ocorriam, simultaneamente, em resposta ao aumento populacional de moscas. Convencionou-se o termo "resposta", que poderia ser "atrasada", se os danos ocorressem após pico populacional, ou "imediata", se os danos coincidissem com este pico.

Registros das temperaturas máxima, mínima e média, da precipitação pluvial e da umidade relativa foram obtidos em estação meteorológica, distante $30 \mathrm{~km}$ das áreas experimentais. Análise de correlação múltipla foi utilizada para explicar a influência das variáveis climáticas na flutuação populacional das moscas-das-frutas, com o software estatístico Bioestat 2.0 (Ayres et al., 2000).

\section{RESULTADOS E DISCUSSÃO}

Anastrepha fraterculus (Wiedemann, 1830) foi a espécie predominante e de importância econômica, com 99\% das moscas obtidas em frutos.

Na laranjeira 'Céu', as variáveis climáticas temperatura (máxima, mínima e média), precipitação pluvial e umidade relativa influenciaram o número de moscas-dasfrutas/armadilha McPhail por dia de exposição (MAD) $(\mathrm{MAD}=-13,41+0,03 \mathrm{~mm}-73,55 \mathrm{Tmax}-74,90 \mathrm{Tmin}+$
148,47Tm + 0,09UR; em que: $\mathrm{R}^{2}=0,4883 ; \mathrm{p}=0,0062$ ). A precipitação foi mais importante e apresentou coeficiente de correlação parcial $(p)=0,0196$. Entretanto, não foi verificada influência dessas variáveis na população de moscas $(\mathrm{MAD}=19,71-0,01 \mathrm{~mm}-0,64 \mathrm{Tmax}+$ $0,12 \mathrm{Tmin}+0,34 \mathrm{Tm}-0,13 \mathrm{UR}$; em que: $\mathrm{R}^{2}=0,4922 ; \mathrm{p}$ $=0,0831$ ) em tangor 'Murcott'.

As diferenciadas situações de coleta dos dados podem explicar essas diferentes significâncias. Em 2003, as chuvas foram consideradas normais para a região; entretanto, no primeiro quadrimestre de 2004, ocorreu severa estiagem. Desta forma, pode-se explicar o fato de a precipitação ter-se destacado no contexto das outras variáveis climáticas. Em outros estudos, essa variável não foi considerada significativa (Chiaradia et al., 2004). A associação entre a precipitação pluvial e o MAD pode ser explicada, em laranjeira 'Céu', em função da época da realização do experimento. No verão e no outono, essa variável tem importância para manutenção da umidade do solo, aumentando a viabilidade das pupas e a emergência de adultos (Aluja, 1994).

Em laranjeira 'Céu', observou-se que o pico populacional de A. fraterculus ocorreu de 18 a 24 de fevereiro de 2003, coincidindo com a maior precipitação pluvial (130 mm); em 2004, esse pico foi constatado de 12 a 19 de fevereiro, menor que no ano anterior. Entretanto, o mesmo padrão de variação foi constatado. Os danos aos frutos da variedade 'Céu' foram menores, em 2004 (Tabela 1), por causa, provavelmente, dessa menor população de moscas-das-frutas.

No tangor 'Murcott', a estiagem, associada às condições de temperaturas inferiores nos meses de amostragens, pode explicar o baixo número de insetos nos pomares. No entanto, em floresta semidecídua, no Estado de Mato Grosso do Sul, Brasil, os maiores picos populacionais de Anastrepha, com exceção de Anastrepha sororcula Zucchi, 1979, ocorreram em períodos de menor precipitação pluviométrica (Canesin \& Uchôa-Fernandes 2007). Os resultados desta pesquisa sugerem que os fatores climáticos não foram os únicos determinantes da variação populacional de $A$. fraterculus. É interessante considerar que outros fatores, como a disponibilidade de alimento, também podem exercer in-

Tabela 1. Percentagem acumulada de danos de A. fraterculus em laranjas 'Céu' e em tangores 'Murcott' no período de maturação, na região do vale do rio Caí, Rio Grande do Sul

\begin{tabular}{lcc}
\hline Anos & Espécies & Danos Acumulados (\%) \\
\hline \multirow{2}{*}{2003} & Laranjeira 'Céu' & 24,1 \\
& Tangor 'Murcott' & 10,1 \\
\hline \multirow{2}{*}{2004} & Laranjeira 'Céu' & 2,3 \\
& Tangor 'Murcott' & 3,4 \\
\hline
\end{tabular}


fluência na área amostrada e não apenas as condições climáticas (Alberti et al. 2012).

Os danos aos frutos da laranjeira 'Céu' foram verificados a partir de 20 de março de 2003 e coincidiram com a mudança de coloração dos frutos. Em 2004, os danos coincidiram com o início da maturação. Em nenhum dos casos, o pico populacional coincidiu com o pico dos danos (Figura 1-A). Mangas semimaduras ou maduras foram preferidas por adultos de Bactrocera dorsalis (Hendel, 1912), em relação às verdes, em função da coloração mais atrativa (Rattanapun et al. 2009). Entretanto, a coloração não foi importante na escolha do substrato para oviposição de A. fraterculus e, sim, as características físico-químicas (Gregorio et al. 2010). No entanto, devemos considerar que, em condições de campo, essas condições ocorrem de forma simultânea. A mudança de coloração coincide com as mudanças físico-químicas da polpa do fruto e condicionam a preferência para oviposição e dano. Destaque deve ser dado para a presença de glândulas com óleos essenciais alelopáticos, na casca de ambos os cultivares de citros, apontadas como responsáveis pela inviabilidade de ovos e mortalidade de larvas de Anastrepha suspensa (Loew, 1862) (Greany et al., 1983). Isto impediria o desenvolvimento larval e o consequente dano no início da maturação, por causa das elevadas concentrações dessa substância, nos frutos, nesse estádio fenológico.

Em laranjeira 'Céu', no ano de 2003, observaram-se correlações significativas do número MAD com os danos aos frutos, em função da população e do estádio de maturação, determinado por meio da escala de colora-
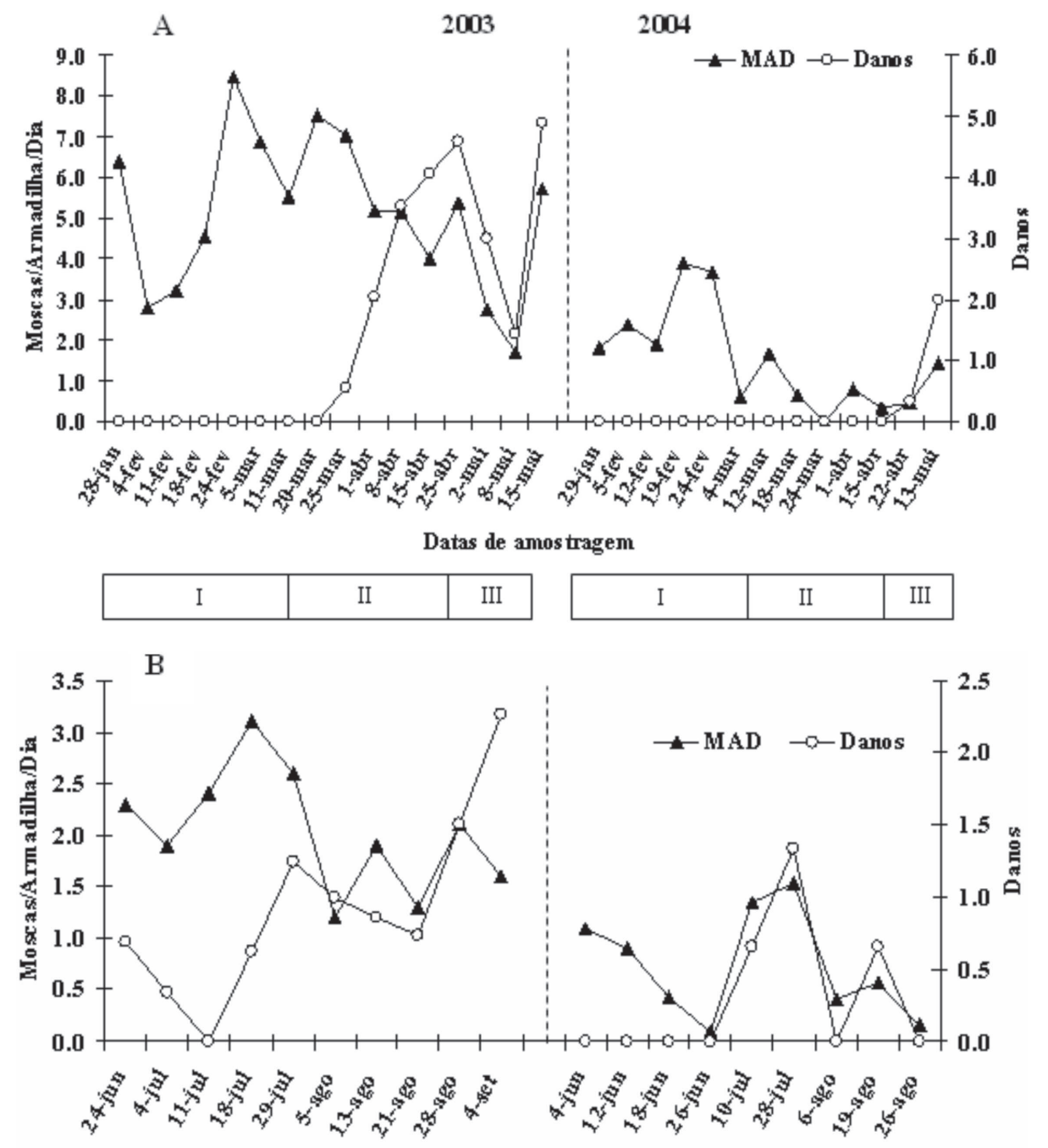

Datas de amostragem

\begin{tabular}{|l|l|l|l|l|l|}
\hline I & II & III \\
\hline
\end{tabular}

Figura 1. Número de moscas/armadilha/dia e danos provocados (\%) em laranjas 'Céu' (A) e tangores 'Murcott' (B), durante a fase de maturação. As barras indicam a mudança de coloração dos frutos: I - frutos verdes; II - mudança de coloração e III - frutos de coloração amarela intensa (maturação).

Rev. Ceres, Viçosa, v. 61, n.5, p. 637-642, set/out, 2014 
ção. Período de 25 dias, a partir da constatação do maior número de moscas, foi necessário para o aparecimento dos danos nos frutos, ou seja, uma resposta atrasada (Figura 1-A). No caso da maturação, a resposta foi imediata; no mesmo momento em que foi constatado o maior número de moscas, também ocorreram os maiores danos aos frutos. Assim, os danos coincidiram no tempo com a maior população. Em 2004, a ocorrência da estiagem interferiu nessa resposta, pela diminuição da população de moscas-das-frutas nos pomares. Foi possível a constatação da resposta imediata, apenas na maturação (Tabela 2).

Em tangor 'Murcott', o comportamento foi semelhante (Figura 1-B). Quando as amostragens foram iniciadas, em 2003, já havia danos em frutos verdes. Esse fato levou à antecipação das amostragens, em 2004, quando os danos foram verificados logo no início da fase de mudança de coloração. Os danos aos frutos, em tangor 'Murcott', por A. fraterculus, em 2003, foram considerados atrasados em sete dias. Este comportamento foi observado até a ocorrência de nova estiagem, na semana seguinte ( 29 de julho), quando a correlação entre população e danos deixou de existir. Isso sugere interferência da precipitação pluvial na ocorrência dos danos. No período de maturação, não ocorreram associações entre população de moscas e danos. Entretanto, em 2004, a resposta dos danos à população de moscas foi imediata, tanto na fase de mudança de coloração quanto na de maturação (Tabela 2).

Isto evidenciaria diferenças em relação à aceitação, adequação ou desenvolvimento das moscas, conforme a espécie de citros disponível e o grau de desenvolvimento e de coloração dos frutos. Esta característica pode estar relacionada com comprimentos de onda, em que as cores violeta, azul, verde e amarela (380 a $570 \mathrm{~nm}$ ) foram preferidas por Anastrepha obliqua (Macquart, 1835) (López-Guillén 2009). Tendências semelhantes foram observadas, em estudos de laboratório e de campo, com Anastrepha ludens (Loew, 1873), atraída para amarelo e verde (Robacker et al., 1990; Robacker, 1992.). A. suspensa mostrou preferência por cor laranja (580-590 nm) (Greany et al., 1978). Neste estudo, embora não quantificado o comprimento de onda, as cores da faixa do amarelo à laranja pareceram ser mais atrativas para A. fraterculus.

A população de A. fraterculus estimada como capaz de causar dano de $1 \%$ aos frutos nos períodos de mudança de coloração e de maturação, variou de acordo com o cultivar. Para laranjeira 'Céu', o índice MAD foi de 1,75 a 0,80; para tangor 'Murcott', variou de 2,83 a 1,16 (Tabela 2). Até o início do período de maturação, em 2004, não foram registrados danos aos frutos, em laranjeira 'Céu', por causa do baixo número de moscas-dasfrutas nos pomares (Figura 2-A).

Os maiores valores de MAD ocorreram na fase de mudança de coloração dos frutos, indicando que o monitoramento e o controle devem ocorrer nesse período, apesar de danos imediatos não serem evidentes. Nesse período, ocorre a maior tolerância dos frutos aos sintomas de danos e o dano é mais elevado, de 1,75 MAD para laranjeira 'Céu' e de 2,83, para tangor 'Murcott'. Considerando-se o fato de os danos ocorrerem de forma atrasada, em condições de campo, a maior preocupação de controle da mosca-das-frutas deve ser na fase de maturação. Nessa fase, as respostas são imediatas e um baixo índice MAD já pode comprometer a produção; entretanto, não é nessa fase que se observa o pico populacional.

Tabela 2. Danos (d) nos frutos em resposta ao número de moscas-das-frutas/armadilha/dia (MAD) e população de moscas para ocasionar $1 \%$ de dano (ND) em laranjas 'Céu' e tangor 'Murcott' na região do vale do rio Caí, RS

\begin{tabular}{|c|c|c|c|c|c|c|}
\hline \multicolumn{7}{|c|}{ Laranja 'Céu' } \\
\hline Anos & Períodos* & Respostas & Equações & $\mathbf{r}$ & $\mathbf{p}$ & ND \\
\hline \multirow{2}{*}{2003} & I a II & Atrasada (25 dias) & $\mathrm{d}=0,02+0,56 \mathrm{MAD}$ & 0,8433 & 0,0715 & 1,75 \\
\hline & III & Imediata & $\mathrm{d}=0,36+0,80 \mathrm{MAD}$ & 0,9017 & 0,0135 & 0,8 \\
\hline \multirow{2}{*}{2004} & I a II & —** & —** & —** & —** & —** \\
\hline & III & Imediata & $\mathrm{d}=-0,56+1,82 \mathrm{MAD}$ & 0,9998 & 0,0130 & 0,85 \\
\hline \multicolumn{7}{|c|}{ Tangor 'Murcott' } \\
\hline Anos & Períodos & Respostas & Equações & $\mathbf{r}$ & $\mathbf{p}$ & ND \\
\hline \multirow{2}{*}{2003} & I a II & Atrasada (7 dias $* * *)$ & $\mathrm{d}=-1,92+1,03 \mathrm{MAD}$ & 0,9958 & 0,0578 & 2,83 \\
\hline & III & Nenhuma & $\mathrm{d}=1,37+0,19 \mathrm{MAD}$ & $-0,8512$ & 0,7498 & 1,94 \\
\hline \multirow{2}{*}{2004} & I a II & Imediata & $\mathrm{d}=-0,33+0,75 \mathrm{MAD}$ & 0,7679 & 0,0447 & 1,77 \\
\hline & III & Imediata & $\mathrm{d}=-0,18+1,01 \mathrm{MAD}$ & 0,9442 & 0,0532 & 1,16 \\
\hline
\end{tabular}

* I - frutos verdes; II - mudança de coloração e III - frutos de coloração amarela intensa (maturação);

** sem dano nesta fase;

*** período de 24 de junho a 29 de julho. 


\section{CONCLUSÕES}

A infestação dos frutos de citros por Anastrepha fraterculus está relacionada com a espécie e o cultivar de citros e com fatores climáticos, principalmente com a precipitação pluvial.

O monitoramento da população dessa mosca-das-frutas é importante na determinação da infestação na colheita, desde a fase anterior à plena mudança da coloração dos frutos.

A laranjeira "Céu" apresenta maior susceptibilidade aos danos de A. fraterculus do que o tangor "Murcott", sendo a fase de maturação a mais crítica para ambas as espécies.

\section{REFERÊNCIAS}

Alberti S, Bogus GM \& Garcia FRM (2012) Flutuação populacional de moscas-das-frutas (Diptera, Tephritidae) em pomares de pessegueiro e maracujazeiro em Iraceminha. Biotemas, 25:53-58.

Aluja M (1994) Bionomics and management of Anastrepha. Annual Review of Entomology, 39:155-178.

Ayres M (2000) Bioestat 2.0: aplicações estatísticas nas áreas das ciências biológicas e médicas. Brasília, CNPq. 272p.

Canesin A \& Uchoa-Fernandes MA (2007) Faunistic study and populational fluctuation of fruit flies (Diptera, Tephritidae) in a fragment of semideciduous forest in Dourados, Mato Grosso do Sul, Brazil. Revista Brasileira de Zoologia, 24:185-190.

Chiaradia LA, Milanez JM \& Dittrich R (2004) Flutuação populacional de moscas-das-frutas em pomares de citros no oeste de Santa Catarina, Brasil. Ciência Rural, 34:337-343.

Dg Colour Ltd (1996) Munsell Conversion Program: Versão 4.0.1 para PC baseado em Windows.

Greany PD, Burditt Jr AK, Agee HR \& Chambers DL (1978) Increasing effectiveness of visual traps for the Caribbean fruit fly, Anastrepha suspensa (Diptera: Tephritidae), by use of fluorescent colors. Entomologia Experimentalis et Applicata, 23:20-25.

Greany PD, Syter DS, Davis PL, Shaw PE \& Chambers DL (1983) Biochemical resistance of citrus to fruit flies: demonstration and elucidation of resistance to the Caribbean fruit flies, Anastrepha suspensa. Entomologia Experimentalis et Applicata, 34:40-50.

Gregorio PLF, Sant'Ana J \& Redaelli LR (2010) Percepção química e visual de Anastrepha fraterculus (Diptera, Tephritidae) em laboratório. Iheringia, 100:128-132.

Lopez-Guillen G, Virgen A \& Rojas JC (2009) Color preference of Anastrepha obliqua (Diptera, Tephritidae). Revista Brasileira de Entomologia, 53:157-159.

Malavasi A \& Morgante JS (1981) Adult and larval population fluctuation of Anastrepha fraterculus and its relationships to host availability. Environmental Entomology, 10:275-278.

Paiva PEB (2004) Moscas-das-frutas em citros: densidade de armadilhas para monitoramento, efeito do $\mathrm{pH}$ na atração e determinação do nível de controle. Dissertação de Mestrado. Escola Superior de Agricultura Luiz de Queiroz, Piracicaba. 48p.

Pedigo LP (1996) Entomology and pest management. New Jersey, Prentice-Hall, 679p.

Rattanapun W, Amornsak W \& Clarke A (2009) Bactrocera dorsalis preference for and performance on two mango varieties at three stages of ripeness. Entomologia Experimentalis et Applicata, 131:243-253.
Robacker DC (1992) Effects of shape and size of colored traps on attractiveness to irradiated, laboratory-strain Mexican fruit flies (Diptera: Tephritidae). Florida Entomologist, 75:230-240.

Robacker DC, Moreno DS \& Wolfenbarger DA (1990) Effects of trap color, height, and placement around trees on capture of Mexican fruit flies (Diptera: Tephritidae). Journal of Economic Entomology, $83: 412-419$.

Salles LAB (1995) Bioecologia e controle da mosca-das-frutas sul-americana. Pelotas, Embrapa CPACT. 58p.

Scoz PL, Botton M, Garcia MS \& Pastori PL (2006) Avaliação de atrativos alimentares e armadilhas para o monitoramento de Anastrepha fraterculus (Wiedemann, 1830) (Diptera: Tephritidae) na cultura do pessegueiro (Prunus persica (L.) Batsh). Idesia, 24:7-13.

Sequeira R, Millar L \& Bartels D (2001) Identification of susceptible areas for the establishment of Anastrepha spp. fruit flies in the United States and analysis of selected pathways. [S.1.], USDA. 28p.

Zucchi RA (2000) Taxonomia. In: Malavasi A, Zucchi RA Moscas-dasfrutas de importância econômica no Brasil: conhecimento básico e aplicado. Ribeirão Preto, Holos, p.13-24.

Rev. Ceres, Viçosa, v. 61, n.5, p. 637-642, set/out, 2014 